BULLETIN OF THE

AMERICAN MATHEMATICAL SOCIETY

Volume 80, Number 5, September 1974

\title{
ON IDEALS OF COMPACT OPERATORS
}

\author{
BY J. S. MORRELL, ${ }^{1}$ M. S. HSIEH AND J. R. RETHERFORD ${ }^{2}$
}

Communicated by Robert Bartle, January 7, 1974

In [1] Brown, Pearcy and Salinas give an affirmative answer to the following question: Given a compact operator $T$ on a separable Hilbert space $H$, is there an ideal $\Lambda(T)$ containing $T$ and different from the ideal $K$ of all compact operators? Their construction relies on some ideas of Von Neumann-Calkin [2] and is rather complicated.

The purpose of this brief note is to show that the existence of such a $\Lambda(T)$ follows from elementary properties of the $s$-numbers of $T$. Recall that the $s$-numbers, $\left(s_{n}(T)\right)$, are the eigenvalues of $\left(T T^{*}\right)^{1 / 2}$ arranged in decreasing order and counting multiplicities. We list the three properties we need:

(1) If $S, T \in K$, then $s_{n+m}(S+T) \leqq s_{n}(S)+s_{m}(T)$.

(2) If $R, S, T \in L$, then $s_{n}(R S T) \leqq\|R\| s_{n}(S)\|T\|$. Here $L$ denotes the space of all bounded linear operators on $H$.

(3) If $T \in K$, then there are orthonormal sets $\left(f_{n}\right)$ and $\left(y_{n}\right)$ in $H$ such that $T=\sum_{n=1}^{\infty} s_{n}(T) f_{n} \otimes y_{n}$.

We also use the following fact concerning real sequences:

(4) If $\left(\beta_{n}\right)$ is a nonnegative sequence of real numbers increasing to $\infty$ with $1\left|\beta_{n} \in c_{0}\right| \bigcup_{p>0} l_{p}$, then there is a positive sequence $\left(\alpha_{n}\right) \in l_{1}$ such that $\sum_{n=1}^{\infty} \alpha_{n} \beta_{n}=+\infty$ and $\left(\beta_{n} \alpha_{n}\right)$ is decreasing. Also, there is a decreasing null sequence $\left(\gamma_{n}\right)$ such that $\sum_{n=1}^{\infty} \gamma_{n} \alpha_{n} \beta_{n}=+\infty$.

The construction of $\Lambda(T)$. Let $\sigma_{p}=\left\{T \in K: \sum_{n=1}^{\infty} s_{n}(T)^{p}<+\infty\right\}$. It is well known and easy to prove that $K \backslash \bigcup_{p>0} \sigma_{p} \neq \varnothing$. (For a study of these important ideals see [3] and [4].) Thus we may suppose, for our purpose, that $T \in K \backslash \bigcup_{p>0} \sigma_{p}$. Then $\beta_{n}=1 / s_{n}(T)$ increases to $\infty$ and $1 / \beta_{n} \in$ $c_{0} \backslash \bigcup_{p>0} l_{p}$. Let $\left(\alpha_{n}\right)$ be as in (4) and let

$$
\Lambda(T)=\left\{S \in L: \sum_{n=0}^{\infty} s_{n}(S) \alpha_{n} \beta_{n}<+\infty\right\} .
$$

It follows from (4) and the definition of $\left(\beta_{n}\right)$ that $T \in \Lambda(T)$.

AMS (MOS) subject classifications (1970). Primary 47B05, 41A45.

${ }^{1}$ Research supported by a Faculty Council Research Grant from the University of Southern Mississippi, Hattiesburg, Mississippi 39401.

${ }^{2}$ Research supported by NSF-GP 34193. 
If the rank of $S$ is finite, then $s_{n}(S)=0$ for large enough $n$ and so $\Lambda(T)$ contains the finite rank operators (this fact also follows from deeper considerations).

If $R, S \in \Lambda(T)$ then by (1) and (4)

$$
\alpha_{2 n} \beta_{2 n} s_{2 n}(R+S) \leqq \alpha_{n} \beta_{n} s_{n}(R)+\alpha_{n} \beta_{n} s_{n}(S),
$$

and similarly for $\alpha_{2 n-1} \beta_{2 n-1} s_{2 n-1}(R+S)$. Thus $\Lambda(T)$ is a linear space. Also, it follows from (2) that $\Lambda(T)$ is closed under left and right composition by bounded linear operators.

Let $T_{0}=\sum \gamma_{n} f_{n} \otimes y_{n}$, where $\left(\gamma_{n}\right)$ is as in (4) and $\left(f_{n}\right)$ and $\left(y_{n}\right)$ are determined by (3). Then $s_{n}\left(T_{0}\right)=\gamma_{n+1}$ and so by (4) $T_{0} \in K \backslash \Lambda(T)$.

Using the recent results [5] and [6] it is easy to generalize the above construction to include many other classes of Banach spaces.

Recall that the $n$th approximation numbers, $\alpha_{n}(T)$, of $T$ are given by

$$
\alpha_{n}(T)=\inf \{\|T-A\|: \operatorname{rank} A \leqq n\} .
$$

For Banach spaces $E, F$ we say $T \in l_{p}(E, F)$ if $\sum_{n=1}^{\infty} \alpha_{n}(T)^{p}<+\infty$. These generalized ideals have been studied by Pietsch and others. The only fact we need here is the following [5]: If $K(E, F)=l_{p}(E, F)$ for some $p>0$, then $\min (\operatorname{dim} E, \operatorname{dim} F)<+\infty$. Here $K(E, F)$ denotes the compact operators from $E$ to $F$. We also recall the following definition [6]: Two Banach spaces $E$ and $F$ form a Bernstein pair if for any positive, decreasing null sequence $\left(b_{n}\right)$ there is a $T \in K(E, F)$ such that

$$
0<\inf _{n} \frac{\alpha_{n}(T)}{b_{n}} \leqq \sup _{n} \frac{\alpha_{n}(T)}{b_{n}}<+\infty .
$$

Let $\mathscr{K}$ denote the ideal of all compact operators between arbitrary Banach spaces and let $T \in \mathscr{K}$.

THEOREM. There exists a complete quasi-normed ideal $\Lambda(T)$ such that $\Lambda(T)(E, F) \neq K(E, F)$ whenever $\langle E, F\rangle$ forms a Bernstein pair.

Indeed, by the result of Pietsch, we may assume that $\left(\alpha_{n}(T)\right) \in$ $c_{0} \mid \bigcup_{p>0} l_{p}$. Let $\beta_{n}=1 / \alpha_{n}(T)$ and let $\alpha_{n}, \gamma_{n}$ be as in (4) above. Let

$$
\Lambda(T)=\left\{S \in \mathscr{K}: \sum_{n=1}^{\infty} \alpha_{n}(S) \alpha_{n} \beta_{n}<+\infty\right\} .
$$

Since the approximation numbers have properties (1)-(3), $\Lambda(T)$ is ideal. It is easy to show that under the quasi-norm

$$
\rho(S)=\sum_{n=1}^{\infty} \alpha_{n}(S) \alpha_{n} \beta_{n},
$$


$\Lambda(T)$ is complete. If $\langle E, F\rangle$ is a Bernstein pair, then by (5) there is an $S \in K(E, F)$ such that $\inf _{n}\left(\alpha_{n}(S) / \gamma_{n}\right)>0$ and thus, by (4), $S \notin \Lambda(T)(E, F)$.

We remark that all classical Banach spaces form Bernstein pairs. In particular $\left\langle L_{p}(\mu), L_{q}(\gamma)\right\rangle$ is a Bernstein pair for all $1 \leqq p, q \leqq \infty$ and measures $\mu, \gamma[6]$.

\section{BIBLIOGRAPHY}

1. A. Brown, C. Pearcy and N. Salinas, Ideals of compact operators on Hilbert space, Michigan Math. J. 18 (1971), 373-384. MR 45 \#909.

2. J. W. Calkin, Two-sided ideals and congruences in the ring of bounded operators in Hilbert space, Ann. of Math. (2) 42 (1941), 839-873. MR 3, 208.

3. I. C. Gohberg and M. G. Krein, Introduction to the theory of linear nonself-. adjoint operators in Hilbert space, "Nauka", Moscow, 1965; English transl., Transl. Math. Monographs, vol. 18, Amer. Math. Soc., Providence, R.I., 1969. MR 36 \#3137; 39 \#7447.

4. R. Schatten, Norm ideals of completely continuous operators, Ergebnisse der Math. und ihrer Grenzgebiete, N.F., Heft 27, Springer-Verlag, Berlin, 1960. MR 22 \#9878.

5. A. Pietsch, The ideals $l_{p}(E, F), 0<p<\infty$ are small (to appear).

6. C. Hutton, J. S. Morrell and J. R. Retherford, Approximation numbers and Kolmogoroff diameters of bounded linear operators, Bull. Amer. Math. Soc. 80 (1974), 462-466.

Department of Mathematics, University of Southern Mississippi, Hattiesburg, MisSISSIPPI 39401

Department of Mathematics, Louisiana State University, Baton Rouge, LOUISIANA 70803 